\title{
Programa de Atención Psicológica en Neonatología: Experiencia del Hospital Clínico San Carlos de Madrid
}

\section{Psychological Care Program in Neonatology: The Hospital Clínico San Carlos's experience}

\author{
Isabel Cuéllar Flores y Mercedes Valle Trapero \\ Hospital Universitario Clínico San Carlos de Madrid, España
}

\begin{abstract}
Resumen: En España son muy pocos los hospitales donde se desarrollan programas específicos de psicología perinatal en Neonatología, a pesar de las recomendaciones de los expertos y de las guías de práctica clínica que señalan que es necesario proporcionar apoyo psicológico a las familias y a los profesionales. El Hospital Universitario Clínico San Carlos de Madrid cuenta con un programa pionero y específico de psicología perinatal en Neonatología desde hace más de dos décadas. En el presente trabajo se describen las características del programa. Palabras clave: psicología perinatal, psicología neonatal, prematuridad, salud mental materna, Atención Temprana.
\end{abstract}

\begin{abstract}
Very few Spanish hospitals have specific psychological care programs in neonatology, despite experts' recommendations and the clinical practice guidelines suggesting the need to provide psychological support to families and healthcare staff. Hospital Clínico San Carlos of Madrid implemented a pioneer, specific perinatal psychology program in neonatology more than two decades ago. This article describes the characteristics of the program. Keywords: perinatal psychology, neonatal psychology, prematurity, maternal mental health, Early Care.
\end{abstract}

Es nuestra responsabilidad como cuidadores, científicos, políticos, profesionales de salud mental, y ciudadanos responsables que cada bebé tenga la oportunidad de aprender a amar y que cada padre y madre reciba el apoyo necesario para que esto suceda.

Ruth Feldman

Las autoras son psicólogas clínicas en el Hospital Universitario Clínico San Carlos de Madrid.

Para cualquier información sobre este artículo debe ponerse en contacto con la primera autora en el e-mail: isabel.cuellar@salud.madrid.org

(cc) BY-NC-ND Este es un artículo Open Access bajo la licencia CC BY-NC-ND. 
La psicología neonatal es "el estudio científico de los procesos biopsicosociales y comportamentales que influyen en la salud, enfermedad y el cuidado sanitario del neonato pretérmino (y a término) durante sus primeros [...] días de vida, y la relación entre esos procesos y el posterior desarrollo" (trad. propia de Adamson-Macedo, 2004). Es por ello que incluye también los cambios emocionales y psicológicos que se producen durante el proceso de maternidad y paternidad en la pareja de padres. Tiene como marco de referencia la Psicología del Desarrollo y la Psicología Perinatal, la Atención Temprana y la Psicología de la Salud, con características propias y diferentes del contexto de salud mental.

En 2010 hubo 486.575 nacimientos (Instituto Nacional de Estadística, 2010) y se registró en España una tasa de nacimientos pretérmino del 7.45\% (Ministerio de Sanidad, 2014b). En el año 2015 nacieron un total de 3590 bebés de menos de 32 semanas de gestación (Instituto Nacional de Estadística, 2015). Además se calcula que entre 500 y 1500 recién nacidos por año en España presentarán una encefalopatía hipóxico-isquémica significativa al nacer (García-Alix y González de Dios, 2010). Éstas y otras condiciones clínicas de grave riesgo biológico requieren hospitalización en Neonatología (Ministerio de Sanidad, 2014b). Además en 2010 se registraron 400 recién nacidos fallecidos (Ministerio de Sanidad, 2014b). Las madres de estos recién nacidos hospitalizados presentan mayor frecuencia de depresión posparto, estrés postraumático y otros síntomas de distrés psicológico (Brandon et al., 2011; Friedman et al., 2013; González-Serrano, 2009), y se ha descrito que entre el 20 y $30 \%$ experimentan un trastorno mental diagnosticable durante el primer año después del parto (Hynan, Mounts y Vanderbilt, 2013). Es por ello que la inclusión de psicólogos en las Unidades de Neonatología es una práctica cada vez más frecuente en Estados Unidos y Reino Unido (Hynan y Hall, 2015; Hynan et al., 2015) y se ha demostrado que permite detectar mejor la psicopatología maternal, promover el ajuste psicológico de los padres y mejorar las interacciones entre los padres y el equipo sanitario (Hynan et al., 2015; Patterson, 2013). La psicoterapia, además, en las Unidades de Cuidados Intensivos Neonatales (UCIN) tiene resultados beneficiosos (Shaw et al., 2013).

En España, sin embargo, son aún muy pocos los hospitales donde se desarrollan programas específicos de psicología perinatal en Neonatología, con algunas excepciones (Olza, Palanca, González-Villalobos, Malalana y Contreras, 2014, aunque actualmente no se encuentre en funcionamiento o, García-Villanova, Cuéllar-Flores, Eimil y Palacios, 2013; Tarragó, 2003. Y ello a pesar de las recomendaciones de los expertos y de las guías de práctica clínica en Neonatología en nuestro país, que señalan que es necesario proporcionar apoyo a las familias, tanto psicológico, como psiquiátrico, en caso necesario, y social (Ministerio de Sanidad, 2014b). Este tipo de apoyo, afirman, se debe extender también al personal sanitario de las unidades de neonatología, dado que el hecho de experimentar de forma reiterada situaciones de sufrimiento y muerte puede afectarles negativamente (Tejedor et al., 2013).

Uno de los hospitales que cuenta con un programa específico de psicología perinatal en Neonatología es el Hospital Universitario Clínico San Carlos de Madrid (HUCSC), cuya Unidad de Neonatología incluyó a una Psicóloga Clínica en su equipo, adscrita a la Unidad de Neonatología, en 1989. Se creó entonces la Unidad de Psicología Clínica-Atención Temprana (UPC-AT), que cuenta en la actualidad con una Psicóloga Clínica que trabaja en coordinación con neonatólogos y dos trabajadoras sociales y que ha desarrollado hasta el momento dos programas complementarios y coordinados con los de control prospectivo de poblaciones de Alto Riesgo (Arizcun, Valle y Arrabal, 2003; Arrabal, 1999): el Programa de Atención Psicológica en Neonatología y el Programa de Atención Temprana centrado en la Familia. El segundo de ellos ha sido descrito en varios trabajos previos (Valle, 2010; Valle, Mateos y Gútiez, 2012; Valle, Sierra, Palacios, Ormazábal y Moro, 2014) y está orientado al seguimiento psicológico después del alta y hasta al menos los dos años de vida de los bebés que han sido hospitalizados en UCIN con factores de alto riesgo para el desarrollo psicomotor y psicoemocional y de sus familias.

En el presente trabajo se describe el modelo de trabajo y el funcionamiento del Programa de Atención Psicológica en Neonatología de esta Unidad, explicando el contexto teórico y práctico que desarrolla en el ámbito de la salud mental maternal, paternal y vincular.

Para dar una idea del volumen asistencial, la Unidad de Neonatología cuenta con 11 camas de UCIN y 12 camas de Cuidados Medios. Se trata de un hospital clasificado como 3b, es decir es un hospital de referencia con maternidad y un servicio de pediatría donde se desarrollan la mayor parte de las áreas específicas pediátricas, y se incluye la atención a recién nacidos con edad gestacional inferior a 28 semanas o peso de nacimiento 
inferior a $1000 \mathrm{~g}$ (Blanco, 2004). En el año 2016 un total de 154 recién nacidos precisaron ingreso en la UCIN del HUCSC y 231 en cuidados medios neonatales, siendo la estancia media en cuidados intensivos de 17.6 días y en cuidados medios de 10.02 días. En total 25 bebés de menos de $1500 \mathrm{~g}$ de peso y/o menores de 32 semanas y 9 bebés con Encefalopatía Hipóxico-Isquémica estuvieron hospitalizados en ese año. Se produjeron un total de 10 fallecimientos en menores de un mes de vida. En 2016 se realizaron 86 peticiones de interconsulta a Psicología desde Neonatología y 5 desde la planta de maternidad. De las cuales 27 fueron para el programa de Hospitalización Domiciliaria-Alta precoz.

La filosofía de trabajo de la Unidad de Neonatología se basa en los Cuidados Centrados en el Desarrollo como un programa de estimulación protectora (Arrabal, 1999; Als, 2002), que sitúa al bebé y a la familia en el centro de atención (Als, Duffy, y McAnulty, 1996). Dentro de esta filosofía se considera imprescindible la atención psicosocial, y en particular, la atención psicológica a la unidad recién nacido-padres, pues "la unidad amén de preocuparse de los aspectos puramente biológicos del neonato, tiene un interés muy especial en conocer los problemas de la familia y su entorno" (Valle, 1991, p.51).

\section{Programa de Atención Psicológica en Neonatología del HUCSC}

Este programa se ha ido desarrollando desde los inicios de la creación de la UPC-AT. Incluye varios ámbitos de actuación y subprogramas. Con propósitos explicativos se ha dividido la descripción del programa en cuatro ámbitos (Valoración y prevención, Intervención, Coordinación y enlace, y Docencia e investigación). No obstante los dos primeros no siempre están tan delimitados, pues consideramos que la valoración y diagnóstico es un proceso y no un hecho aislado. Además, en la prevención primaria, secundaria y terciaria se solapan algunos objetivos y técnicas de intervención. Hasta el mismo acto de presentarse como psicóloga supone ya alguna clase de intervención y puede ejercer algún efecto que debemos tener en cuenta.

\section{Valoración Psicológica y Prevención}

El nacimiento y hospitalización del bebé puede provocar sentimientos de extrañeza y fragilidad, una enorme exigencia de disponibilidad, y el término brusco de la sensación de fusión con éste (López, 2014). En ocasiones aparecen sentimientos de culpa (por "dejar" al bebé en el hospital o por atribuirse la responsabilidad de que las cosas no hayan ido como esperaban), hipervigilancia o labilidad emocional. Algunos progenitores parecen encontrarse "disociados", o muestran muy poco impacto afectivo. Todas estas reacciones son frecuentes y no deben ser patologizadas, forman parte del proceso normal de adaptación y afrontamiento de una situación tan estresante como es la hospitalización del propio hijo o hija. Sólo cuando estas reacciones son tan intensas o tan prolongadas en el tiempo que interfieren con la presencia o disponibilidad afectiva de la madre o del padre durante la hospitalización se consideran disfuncionales.

Los objetivos iniciales de la UPC-AT con respecto a la hospitalización en Neonatología son: detectar las necesidades psicológicas de los padres, apoyar a las familias, optimizar el vínculo padres-bebé y asegurarse de que los padres hayan recuperado sus competencias para los cuidados tras el alta.

La prevención y detección temprana de psicopatología y distrés emocional y relacional parental se realiza por parte de todo el equipo (neonatólogos, personal de enfermería y trabajo social) y a su vez se implementa mediante varios protocolos o consensos de atención. Concretamente, a través del programa de Hospitalización Domiciliaria-Alta precoz, el programa de Prevención del Riesgo Social en neonatología (Valle et al., 2012 o Valle et al., 2014) y mediante la derivación a la UPC-AT de todas las familias de recién nacidos con mayor riesgo en su neurodesarrollo, identificados como de Alarma III por la Sociedad Española de Neonatología (Robles, Poo y Poch, 2008). Además, la atención psicológica puede surgir a petición de los neonatólogos, trabajadora social, personal de enfermería y ocasionalmente a petición familiar. Debemos mencionar asimismo el Protocolo de Humanización de los Cuidados del Recién Nacido al final de la vida en el que se incluye la atención psicológica.En la Tabla 1 se muestran los factores de riesgo biológico y psicosocial que suponen la derivación a la UPC-AT. 


\section{Tabla 1. Criterios de derivación a la UPC-AT durante la hospitalización en Neonatología del HUCSC}

Criterios de derivación sistemática

\begin{tabular}{ll}
\hline Factores de Riesgo & Apgar <3 al minuto \\
neurobiológico** & Encefalopatía hipóxico isquémica \\
& Ventilación mecánica desde el nacimiento >24h \\
& Disfunción neurológica persistente o daño cere- \\
bral en pruebas de imagen & Convulsiones neonatales \\
& Malformaciones del SNC \\
& Cromosomopatías \\
& Encefalitis neonatal \\
& Gemelo, si el hermano presenta riesgo neuro- \\
& biológico \\
& Peso < de 1500 gr. ó menos de 32 semanas de \\
& EG. \\
& Cualquier patología que exija intubación > 3 \\
& dias \\
& Convulsiones que ceden difícilmente a la tera- \\
& pia. \\
& Apneas que exigen ayuda respiratoria con in- \\
& tubación. \\
& Síndrome irritativo. Hemisíndrome. Multisín- \\
& drome.
\end{tabular}

\section{Criterios de derivación a demanda}

Síndrome de abstinencia

Bajo peso para la edad gestacional

$\begin{array}{ll}\begin{array}{ll}\text { Factores de Riesgo } \\ \text { psicosocial** }\end{array} & \text { Fallecimiento del bebé } \\ & \text { Padres muy jóvenes } \\ & \begin{array}{l}\text { Trastornos psicopatológicos importantes en los } \\ \text { padres }\end{array}\end{array}$

Falta de presencia de los padres en la UCIN

Embarazadas con amenaza de parto prematuro y reacciones adaptativas

Drogadicción

Violencia de género

Pérdidas perinatales

Reacciones de estrés o adaptativas intensas o que interfieren con los cuidados de los padres

Ovodonación

En el primer contacto con los padres se explicita que se realizan entrevistas con "todas las familias de los bebés hospitalizados en UCIN", para normalizar y reducir el estigma acerca del estrés y malestar parental. Salvo contadas ocasiones los padres aceptan hablar con la psicóloga, y agradecen su presencia y disponibilidad en el servicio. En la primera entrevista se identifican mediante entrevista clínica: 1) el nivel de distrés emocional de los padres, así como posibles indicadores de psicopatología emocional y factores de riesgo vincular; y 2) las capacidades parentales y recursos personales y familiares de esa madre o ese padre. Las fortalezas de los padres pueden incluir la sensibilidad predispuesta biológicamente para la madre (Ministerio de Sanidad, 2014b), la anticipación e identificación de características y necesidades del bebé (Forcada-Guex, Pierrehumbert, Borghini, Moessinger y Muller-Nix, 2006), una red familiar o social que apoye afectiva o instrumentalmente o un buen apoyo mutuo en la pareja de padres. 
La mayoría de los padres y madres presentan un nivel de distrés emocional moderado, con temor a la pérdida y a la discapacidad, dolor psicológico ante la separación y sentimientos de culpa (Acosta-Romo, Cabrera, Basante-Castro y Jurado, 2017). Son infrecuentes los diagnósticos psiquiátricos.

Simultáneamente, y de forma preventiva se ayuda a identificar las señales de estrés y mecanismos de autorregulación de su bebé y se proporciona información sobre conceptos básicos como la edad corregida y los beneficios de la presencia y contacto materno en el desarrollo neurocomportamental y en el inicio del vínculo. Se tiene presente que se trata de bebés prematuros y de padres también prematuros, que no han tenido tiempo de completar el "proceso de anidación psicobiológica" (Jofré y Enríquez, 2002), y se pone nombre a las emociones sentidas, para favorecer la función protectora de la universalización y de la expresión emocional.

Es necesario cumplir para estos padres una "función maternante" que suponga contener y acompañar, señalar y potenciar los recursos y fortalezas de esa madre y de ese padre, que han visto truncados sus deseos y expectativas sobre el nacimiento y sobre su bebé y que se sienten en muchos casos "fuera de lugar" en una UCIN que no es su hogar y en la que ellos no se sienten imprescindibles. Se trata de facilitar que recuperen su "identidad como madre o padre".

Asimismo debe potenciarse la identificación con ese bebé, favoreciendo el "nacimiento psíquico", que no siempre coincide con el nacimiento físico, tejiendo la red de identificaciones, preguntando por la elección del nombre y los parecidos y rescatando la singularidad de ese bebé que es su hijo. Debemos tener en cuenta, asimismo, algunos elementos que pueden requerir una especial atención y sensibilidad, como son los procesos de reproducción asistida o la ovodonación. En estos casos la pareja de padres acude con una historia significativa de pérdidas (ej. duelo genético en casos de ovodonación - o los diversos duelos que supone la infertilidad, el llamado duelo de duelos).

La hospitalización es, como vemos, un momento privilegiado y único para facilitar que la madre y el padre puedan comenzar la vinculación con el recién nacido de forma saludable y no se fije ese período potencialmente traumático.

En la Tabla 2 se muestra un resumen de este apartado.

\section{Tabla 2. Objetivos, técnicas y contenidos de la evaluación y prevención de la UPC-AT durante la hospitalización en Neonatología}

\section{Objetivos y técnicas}

Detección temprana de psicopatología emocional y relacional parental

\section{Contenidos}

Depresión posparto, estrés postraumático y problemas de ansiedad, psicosis posparto

Rechazo y riesgo social

-Capacidades parentales de anticipación e identificación de características y necesidades del bebé

-Capacidades del bebé y semiología de alarma**

Psicoeducación temprana: Información sobre....

Señales de estrés y mecanismos de autorregulación del bebé**

Edad corregida

Sistemas interrelacionados Als

Organización de estados

Beneficios presencia materna e inicio vinculo

AL ALTA:

Contacto con grupos de apoyo

Recomendaciones posturales y ambiente contenedor

Autocuidado (visitas, paseos, sueño...)

Acompañamiento y apoyo psicológico: "nacimiento psicológico" del recién nacido
Nombre y parecidos (red de identificaciones)

Características individuales del bebé (rescatar singularidad) 
Mención especial merecen, además, dos subprogramas en los que colabora la UPC-AT, que son la Hospitalización Domiciliaria-Alta precoz y la Humanización de los Cuidados del Recién Nacido al final de la vida, que se describen a continuación.

Programa de Hospitalización Domiciliaria-Alta precoz. Este programa es uno de los ejemplos paradigmáticos de la interdisciplinariedad en el servicio, y ha recibido el reconocimiento de su importancia y buena práctica (Hernández, Arias, Simón, Cuéllar-Flores, López y Aleo, 2017). La atención psicológica en este programa incluye la valoración de la idoneidad de los padres para marcharse a su casa y asumir todos los cuidados de su hijo estable biológicamente pero con un bajo peso, y la facilitación del proceso para que así sea.

Se valoran diversos aspectos en la pareja de padres: preparación material y psicológica para el alta, apoyo socio-familiar, apoyo mutuo entre los padres, percepción de su hijo o hija y de sí mismos como padres, estado de ánimo y otros síntomas psicopatológicos. Además se pone especial énfasis en favorecer el autocuidado (contacto con grupos de madres, visitas, paseos, sueño...) y la crianza basada en cuidar y proteger (recomendaciones posturales y ambiente contenedor).

Humanización de los Cuidados del Recién Nacido al final de la vida. En la atención al duelo neonatal está incluido todo el personal, y el papel de la psicóloga es favorecer que tanto los padres como el personal puedan transitar esa pérdida. Se trata de facilitar el inicio de un proceso de duelo normal en las familias desde un punto de vista psicológico. Para ello se realizan acciones de acompañamiento y contención, durante la hospitalización cuando es posible, que incluyen a la pareja de padres y, en ocasiones también a los hermanos. Se normalizan las emociones y reacciones, y se favorece que la familia se despida del bebé. Después del fallecimiento se entrega a la familia una caja de recuerdos con pulsera identificativa, una huella del bebé y la cartulina con su nombre. Además la psicóloga realiza llamadas telefónicas de seguimiento a la semana, al mes y 3 meses para transmitir apoyo emocional, detectar necesidad de intervención psicológica adicional y en caso necesario facilitar contacto y derivación a recursos específicos (Friedrichs, Daly y Kavanaugh, 2000). Además se está trabajado en dotar a los profesionales de una guía de actuación y capacitarles para que proporcionen cuidados según las necesidades de cada persona/pareja en duelo, mediante la elaboración de un protocolo de enfermería, actividades formativas para el personal y mediante la creación de un grupo multidisciplinar (con dos enfermeras del equipo) para evaluar y actualizar periódicamente la guía de actuación y que sirva como apoyo para el resto de los profesionales implicados en el proceso.

\section{Intervención Psicológica}

En ocasiones es necesario realizar una intervención psicológica más intensiva, ya sea por la presencia de psicopatología o distrés emocional marcado, por hospitalizaciones más largas o bebés con mayor morbilidad o alteraciones neurobiológicas graves o por indicadores de riesgo vincular (por ejemplo, padres que están poco con su hijo o hija o madres adolescentes con escasa red de apoyo). La mayoría de las intervenciones son psicoterapéuticas de breve duración, ajustándose a las características especiales de este periodo. En cualquiera de los casos la atención que se proporciona debe ser sensible, orientada a las fortalezas e individualizada.

Hay que tener presente que cada familia requiere intervenciones diferenciadas, pero todas tienen en común: 1. El foco en ellos como madre o padre y 2. Facilitar la puesta en marcha de sus recursos y red.

En este momento vital el trabajo psicoterapéutico va a orientarse a las denominadas "interacciones imaginarias" de los padres con ese bebé (Asociación Española de Neuropsiquiatría, 2015), desde la perspectiva de la observación y análisis de las motivaciones y deseos de los padres y del bebé, y de si sus necesidades se ajustan o no, utilizando técnicas específicas (Manzano, 2001).

Las intervenciones psicológicas en las que se transmite a las madres aspectos sobre el desarrollo infantil y sobre cómo proporcionarles una estimulación óptima y responder de forma afectuosa parecen mejorar el ánimo materno, fortalecen la relación madre-bebé y se relacionan con un mejor desarrollo del éste (Rahman, Surkan, Cayetano, Rwagatare y Dickson, 2013). Asimismo, las intervenciones diseñadas para mejorar la salud psico- 
lógica y el bienestar maternal también ejercen un efecto positivo sobre el desarrollo y salud infantil (Rahman et al., 2013). Desde la UPC-AT se interviene sobre ambos aspectos.

Intervención en crisis. La presencia de elevado estrés en las madres ha sido asociado con problemas de regulación en los bebés y con un desarrollo psicomotor y del lenguaje más pobre (Melnyk et al., 2006). El bienestar parental debe ser, por tanto, un importante foco de atención y cuidado en neonatología.

En el contexto de la UPC-AT se realizan intervenciones en crisis y psicoterapia para mejorar la psicopatología y el distress emocional. Se trata de favorecer un afrontamiento saludable desde la óptica del crecimiento ante la adversidad. En ocasiones se busca el restablecimiento emocional: facilitando la comunicación y expresión emocional, mediante la escucha activa (poniendo nombre a las emociones, identificando las necesidades que esas emociones ponen de manifiesto, con reflejos empáticos), la disponibilidad y contención afectiva. A veces la sola posibilidad de saber que se puede contar con un profesional para su cuidado afectivo en caso de necesidad tiene un efecto protector (Dennis y Dowswell, 2013).

También se estimula la movilización de los recursos personales (recuperar el procesamiento cognitivo, habilidades de autocuidado) y sociofamiliares (red de apoyo familiar o social). Se trabaja siempre con las fortalezas (¿qué te ayuda a sobreponerte? ¿qué te ha ayudado en el pasado a afrontar situaciones difíciles?) y se resignifican positivamente los síntomas o las reacciones emocionales o conductuales (por ej. se explica la labilidad emocional materna como el resultado de un estado de sensibilidad afectiva predispuesto biológicamente de forma protectora y saludable, porque facilita la vinculación con el bebé). Partimos de la idea de que son familias sanas que están afrontando una situación difícil, y experimentan "reacciones normales ante una situación anormal".

Programa de Riesgo Social. Cuando se detectan necesidades especiales (socioeconómicas, afectivas, de apoyo sociofamiliar...) en las familias, se movilizan recursos para cubrir al menos parcialmente esas necesidades. La estrecha colaboración con Trabajo Social permite responder a las necesidades socioeconómicas y activar la red de servicios sociales, y otros recursos sociocomunitarios.

Asimismo se ayuda a estar disponibles (por ej. dando información sobre los beneficios del método canguro para su bebé o transmitiendo ideas sobre qué hacer con los afectos intensos) y a disfrutar en el contacto con el bebé (por ej. amplificando la conciencia somática de las sensaciones positivas y placenteras cuando practican el canguro), y se facilita que cada madre y padre sean los maestros de su propio proceso personal.

En ocasiones se convoca a la familia extensa (abuelos) o a los hermanos, sobretodo cuando pueden ejercer un papel protector o suponer un factor de estrés. En las madres adolescentes o solas es muy importante facilitar que la abuela pueda actuar de apoyo afectivo.

Intervención en la Unidad de embarazo de Alto Riesgo. Los obstetras, matronas y neonatólogos piden intervención en situaciones de crisis con madres embarazadas cuando se encuentran hospitalizadas con amenaza de parto prematuro.

El modelo de trabajo es, de nuevo en estos casos, el de la intervención en crisis y el objetivo es reducir el estrés movilizando los recursos de esa madre y ayudándole a recuperar la sensación de cierto control (por ej. dando información sobre cómo es la UCIN o favoreciendo una interacción imaginaria positiva con el bebé).

En la Tabla 3 se resume este apartado.

\section{Coordinación y el Enlace con los Profesionales del Equipo Multidisciplinar}

Una de las ventajas indudables de la UPC-AT es su integración dentro del equipo de neonatología, lo que facilita la disponibilidad ante los problemas del día a día y las necesidades del staff y la posibilidad de mediar tanto entre las familias y el equipo, como entre los diversos estamentos profesionales, así como proporcionar contención, apoyo y escucha. Esto permite participar en reuniones médicas y sesiones clínicas, organizar talleres y actividades formativas (por ejemplo, se han realizado talleres de autocuidado y de abordaje del duelo 


\section{Tabla 3. Objetivos, técnicas y contenidos de la intervención psicológica de la UPC-AT durante la hospitalización en Neonatología}

Objetivos y técnicas

Programa de intervención psicológica

\section{Contenidos}

Intervención en crisis: Restablecimiento emocional: facilitar comunicación y expresión emocional, escucha activa, ofrecer disponibilidad y contención, normalizar emociones

Orientación y activación: movilización de los recursos personales (recuperar procesamiento cognitivo, habilidades de autocuidado) y sociofamiliares (red de apoyo familiar o social).

Iniciar elaboración de pérdidas/duelos sucesivos (nombrarlas, relato integrador)

Intervención para mejorar la interacción temprana madre-bebé: Identificar los recursos del bebé y de la madre/padre

Favorecer funciones parentales de contención y regulación del bebé (estados de conciencia, comportamiento desorganizado)**

Observación de bebes

Facilitar presencia materna
Intervención para mejorar la psicopatología posnatal (DPP, trastornos de ansiedad...)
Terapia psicológica breve

neonatal para el personal) y formar parte de programas de atención interdisciplinar. Como ejemplo de estos últimos está el Programa de Hospitalización Domiciliaria-Alta precoz mencionado previamente, en el que neonatólogos, una enfermera, trabajador social y la psicóloga trabajan estrechamente. Además permite participar en el desarrollo de políticas y protocolos de actuación específicos y en investigación (Shaw y DeMaso, 2009).

Es necesario señalar que el equipo sanitario tiene que soportar una gran presión: afrontar duelos, tomar decisiones difíciles, comunicar malas noticias... no es extraño, por tanto, que en ocasiones se encuentren sobrepasados por la dureza de las situaciones límite que se producen en la UCIN (Friedman, Kessler y Martín, 2014). Por esta razón las funciones esenciales que se plantean desde la UPC-AT con el equipo incluyen la contención y apoyo afectivo de los profesionales, la psicoeducación (Hynan et al., 2015), la facilitación de la comunicación y la mediación (Prasad, 1984). En este marco de trabajo se ha participado en reuniones de equipo para resolver conflictos intraunidad surgidos ante familias complejas, se realizan talleres de autocuidado para personal, acciones de escucha activa individuales con los profesionales y se transmite cierta información que permita empatizar con las familias.

La sola presencia de una psicóloga en el equipo contribuye a mejorar la sensibilidad de los profesionales hacia los aspectos psicológicos y familiares de la atención al recién nacido hospitalizado. Sensibilizar, informar y contribuir a la formación de los distintos profesionales contribuye, asimismo, a favorecer espacios de reflexión y elaboración sobre el mundo psicológico infantil, maternal e interactivo y vincular (Asociación Española de Neuropsiquiatría, 2015).

Además, el intercambio de información en relación al abordaje de la díada madre-bebé es una prioridad y un aspecto fundamental del trabajo en red requerido en este ámbito (Asociación Española de Neuropsiquiatría, 2015). Entre los diversos agentes implicados se encuentran:

- Servicios sanitarios: Neonatología, Enfermería Neonatológica, y en algunos casos Unidad de Cuidados Intensivos Pediátrica, Rehabilitación, Pediatría Hospitalaria, Salud Mental y Ginecología-Obstetricia. 
- Servicios sociales: Trabajo social, y en algunos casos Centros de Apoyo a la Familia o Centros de Atención a la Infancia.

- Otros: asociaciones de atención al duelo perinatal como Umamanita o Red el Hueco de mi vientre.

Es decir, la coordinación y enlace en la UPC-AT supone poner el foco en el equipo sanitario y en su relación con los pacientes y sus familias, lo que redunda en una mejor atención sanitaria y minimiza el burnout y el malestar de los profesionales (Hynan et al., 2015).

\section{Docencia e Investigación}

La UPC-AT es una unidad de referencia en el campo de la Atención Temprana hospitalaria y en el de la psicología perinatal y única en muchos sentidos, por ello numerosos residentes de Psicología Clínica y estudiantes de posgrado se han formado en ella hasta el momento.

En lo que respecta a la investigación, se han mantenido colaboraciones con varias universidades durante estos años. Se han realizado numerosas comunicaciones en congresos, impartido clases en formación de posgrado y se han publicado diversos artículos y capítulos de libros por ej. (Valle et al., 2014).

En el momento actual hay varias líneas de investigación abiertas en prematuridad y otros factores de riesgo neurobiológico neonatal: 1) vínculo, interacción y apego, 2) desarrollo psicomotor y socioafectivo y 3) escolarización temprana en grandes prematuros.

La formación de profesionales en este ámbito tan específico, al igual que la difusión y promoción de la atención psicológica en perinatalidad son funciones de especial relevancia para la unidad.

\section{Discusión y Conclusiones}

El nacimiento de un bebé que tiene que ser hospitalizado produce un elevado nivel de malestar en los padres durante la hospitalización y posteriormente. Además implica una gran fragilidad del recién nacido por ejemplo la tendencia a la desestabilización de sistemas, señales confusas (Als, 2002). Ambos en ocasiones interfieren con la presencia de los padres en la UCIN, con el establecimiento de una interacción y vinculación saludable con su hijo/a y con el desarrollo físico y psicológico del bebé o infante. Por otra parte, y en palabras de uno de los psicólogos perinatales más activos de EEUU, "En la UCIN ideal el apoyo psicosocial a padres y staff serán objetivos igual de importantes que la salud y el desarrollo de los bebés" (Trad. propia, de Hynan y Hall, 2015). La inclusión de la atención psicológica en equipos multidisciplinares sabemos que además contribuye a mejorar la atención médica de los equipos (Brown y Wissow, 2012) y probablemente implique una disminución de los costes globales.

Todas las UCIN deberían disponer de recursos psicosociales para cuidar a las familias de los bebés hospitalizados (Hynan et al., 2015), que forman parte indisociable del bienestar y la salud de los pacientes recién nacidos. Esos recursos psicosociales pueden ser internos o externos a la UCIN e incluir diversos tipos de apoyos (como grupos de padres, profesionales de la salud mental, etc.). Sin embargo, los recursos externos son en general poco utilizados por las familias durante la hospitalización y en los meses siguientes, que están demasiado estresadas y sobrecargadas como para acudir (Goodman, 2009).

En nuestra experiencia, la existencia de la UPC-AT del HUCSC desde hace más de 25 años ha tenido beneficios para las familias y el personal, pues sabemos que las intervenciones psicológicas permiten favorecer la adaptación al estrés materno y paterno, prevenir la aparición de síntomas depresivos, ansiedad y estrés postraumático y mejorar su sensibilidad y capacidad para regular los estados del bebé (interacción y autoeficacia) (Hynan et al., 2015; Patterson, 2013; Shaw et al., 2013). Asimismo favorecen la adaptación al estrés y la capacidad del equipo de Neonatología para contener y acompañar a las familias que atraviesan ese periodo potencialmente traumático (Hynan et al., 2015). Entre las perspectivas futuras de este trabajo se encuentra el disponer de información cualitativa y cuantitativa de los beneficios de las intervenciones psicológicas en las UCIN sobre la adaptación y psicopatología parental y del bebé y promover la investigación sobre los estándares del cuidado y apoyo perinatal en neonatología en nuestro país. 
Disponer, por tanto, de programas de atención psicológica en neonatología como el nuestro, que integra intervenciones en las que se enseña a las madres sobre el desarrollo infantil y sobre cómo estimularles y responder de forma afectuosa, con intervenciones diseñadas para mejorar la salud mental maternal, parece ejercer un efecto mayor en la salud y desarrollo del bebé. Mejor que si los componentes materno e infantil no se integran (Rahman et al., 2013).

\section{Referencias}

Acosta-Romo, M. F., Cabrera-Bravo, N., Basante-Castro, Y. y Jurado, D. (2017). Sentimientos que experimentan los padres en el difícil camino de la hospitalización de sus hijos prematuros. Un aporte al cuidado humanizado. Universidad y Salud, 19(1), 17-25.

Adamson-Macedo, E. N. (2004). Neonatal Psychology: Theories and practice. Neuroendocrinology Letters, 25(1), 9-34.

Asociación Española de Neuropsiquiatría (2015). Psiquiatría perinatal y del niño de 0 a 3 años. Madrid, España: Autor.

Als, H. (2002). Program guide-Newborn Individualized Developmental Care and Assessment Program (NID$C A P)$ : An education and training program for health care professional. Boston, Estados Unidos: Children's Hospital.

Als, H., Duffy, F. H. y McAnulty, G. B. (1996). Effectiveness of individualized neurodevelopmental care in the newborn intensive care unit (NICU). Acta Paediatrica, Supplement, 416, 21-30.

Arizcun, J., Valle, M. y Arrabal, M. C. (2003). El enmarque en los Servicios de Neonatología de los problemas y programas de las discapacidades pediátricas (pp. 299-322). En Curso de Prevención de Deficiencias Materiales. Documentos, ${ }^{\circ}$ 33. Madrid, España: Ministerio de Trabajo y Asuntos Sociales. Real Patronato sobre Discapacidad.

Arrabal, M. C. (Septiembre, 1999). Participación de la familia en los cuidados del recién nacido hospitalizado. En J. Arizcu y J. Rodríguez Alarcón (Presidencia), Neonatología Centrada en la Familia. Mesa Redonda llevada a cabo en el XVIII Congreso de Perinatología, Madrid.

Blanco, D. (2004). Niveles asistenciales y recomendaciones de mínimos para la atención neonatal. Anales de Pediatría, 60(1), 56-64.

Brandon, D., Tully, K., Silva, S., Malcolm, W., Murtha, A., Turner, B. S. y Holditch-Davis, D. (2011). Emotional responses of mothers of late-preterm and term infants. Journal of Obstetric, Gynecologic y Neonatal Nursing, 40, 719-731.

Brown, J. D. y Wissow, L. S. (2012). Rethinking the mental health treatment skills of primary care staff: a framework for training and research. Administration and Policy in Mental Health, 39, 489-502.

Dennis, C. L. y Dowswell, T. (2013). Psychosocial and psychological interventions for preventing postpartum depression. Cochrane Database of Systematic Reviews, 2, CD001134. https://doi.org/10.1002/14651858.C

Forcada-Guex, M., Pierrehumbert, B., Borghini, Moessinger, A. y Muller-Nix, C. (2006). Early Dyadic Patterns of Mother-Infant Interactions and Outcomes of Prematurity at 18 Months. Pediatrics, 118 (1), 107-114. https://soi.org/10.1542/peds.2005-1145

Friedman, S. H., Kessler, R. A. y Martin, R. (2014). Psychiatric help for caregivers of infants in neonatal intensive care. Psychiatric Services, 60, 554. https://doi.org/10.1176/ps.2009.60.4.554

Friedman, S. H., Kessler, A., Yang, S. N., Parsons, S., Friedman, H. y Martin, R. J. (2013) Delivering perinatal psychiatric services in the neonatal intensive care unit. Acta Paediatrica, 102, 392-397.

Friedrichs, J., Daly, M. y Kavanaugh, K. (2000) Follow-up of parents who experience a perinatal loss: facilitating grief and assessing for grief complicated by depression. Illness, Crisis, and Loss, 8, 296-309.

García-Villanova, F., Cuéllar-Flores, I., Eimil, B. y Palacios, I. (2013). El estrés materno en la organización del vínculo madre-bebé prematuro de bajo peso. Clínica Contemporánea, 4, 171-183. https://doi.org/10.5093/ cc2013a14

GAT (2000). Libro blanco de atención temprana. Madrid, España: Ministerio de Sanidad. 
García-Alix, A. y González de Dios, J. (2010). La encefalopatía hipóxico-isquémica en el recién nacido a término ha dejado de ser una entidad huérfana. Implicaciones para la práctica y necesidad de un "código hipotermia". Evidencias en Pediatría, 6(27).

Goodman, J. (2009). Women's attitudes, preferences and perceived barriers to treatment for perinatal depression. Birth, 36(1), 60-69.

González-Serrano, F. (2009). Nacer de nuevo: la crianza de los niños prematuros: Aspectos evolutivos. Atención y acompañamiento al bebé y a la familia. Cuadernos de psiquiatría y psicoterapia del niño y del adolescente, 49, 133-152.

Hernández, M. D. C., Arias, F., Simón, M. J. Cuéllar-Flores, I., López, A. y Aleo, E. (Septiembre, 2017). Implantación de una unidad de hospitalización domiciliaria neonatal para el alta precoz de recién nacidos prematuros y de bajo peso. Póster presentado en el XXVI Congreso de Neonatología y Medicina Perinatal. Zaragoza.

Hynan, M.T. y Hall, S .L. (2015). Introduction: Psychosocial Program Standards for NICU Parents. Journal of Perinatology, Supplement Dec

Hynan, M., Mounts, K. y Vanderbilt, D. (2013). Screening parents of high-risk infants for emotional distress: rationale and recommendations. Journal of Perinatology, 33(10), 748-753.

Hynan, M. T., Steinberg, Z., Baker, L., Cicco, R., Geller, P. A., Lassen, S. y Stuebe, A. (2015). Recommendations for mental health professionals in the NICU. Journal of Perinatology, 35(1), 14-18.

Instituto Nacional de Estadística (2010). Partos. Año 2010. Recuperado de www.ine.es

Instituto Nacional de Estadística (2015). Partos. Año 2015. Recuperado de www.ine.es

Jofré, R. y Enríquez, D. (2002). Nivel de estrés de las Madres con Recién nacidos Hospitalizados en la UCIN Hospital Guillermo Grant. Ciencia y Enfermería, 8, 31-36.

López, M. E. (2014). Sentimientos de las madres con hijos prematuros hospitalizados. Revista IN CRESCENDO - Ciencias de la Salud, 1(2), 263 - 270. DOI: https:// doi.org/10.21895/in+cres+cs.v1i2.352

Manzano, J. (editor) (2001). Las relaciones precoces entre padres e hijos y sus trastornos. Colección infancia y desarrollo. Madrid, España: Necodisne.

Melnyk, B. M., Fischbeck-Feinstein, N., Alpert-Gillis, L., Fairbanks, E., Crean, H. F., Sinkin, R. A., ... Gross, S. J. (2006). Reducing premature infants' length of stay and improving parents' mental health outcomes with the Creating Opportunities for Parent Empowerment (COPE) neonatal intensive care unit program: A randomized controlled trial. Pediatrics, 118, 1414-1437.

Ministerio de Sanidad (2014a). Maternidad y Salud Ciencia, Conciencia y Experiencia. Madrid, España: Autor.

Ministerio de Sanidad (2014b). Unidades de Neonatología. Estándares y recomendaciones de calidad. Madrid, España: Autor.

Olza, I., Palanca, I., González-Villalobos , I., Malalana, A. M. y Contreras, A. (2014). La salud mental del recién nacido hospitalizado: psiquiatría infantil en neonatología. Cuadernos de Medicina Psicosomática, $109,45-49$.

Patterson, C.A. (2013). System level challenges surrounding psychological support for NICU parents. En P. A.Geller (Ed.), Psychologists in the neonatal intensive care unit (NICU). Honolulu, HI, Estados Unidos: American Psychological Association.

Prasad, A. (1984). The role of a liaison psychiatrist in a neonatal intensive care unit. Indian Journal of Psychiatry, 26(1), 79-81.

Rahman, A., Surkan, P. J, Cayetano, C. E., Rwagatare, P. y Dickson, K. E. (2013). Grand Challenges: Integrating Maternal Mental Health into Maternal and Child Health Programmes. PLoS Medicine, 10(5), 1001442. https://doi.org/10.1371/journal.pmed.1001442

Robles, M. C., Poo, M. P. y Poch, M. L. (2008). Atención temprana: recursos y pautas de intervención en niños de riesgo o con retrasos del desarrollo. En J. Narbona y C. Casas (Eds.), Neurología: Protocolos Diagnóstico-Terapéuticos de la Asociación Española de Pediatría. Madrid, España: Asociación Española de Pediatría - Neurología Pediátrica.

Shaw, R. J. y DeMaso, D. R. (2009). Consulta Psiquiatría en Pediatría. En niños y adolescentes con enfermedad orgánica. Buenos Aires, Argentina: Editorial Médica. 
Shaw, R., John, S. N, Lilo, E., Jo, B., Benitez, W., Stevenson, D. y Horwitz, S.M. (2013). Prevention of traumatic stress in mothers of preterm infants: a randomized controlled trial. Pediatrics, 132(4), 1-9.

Tarragó, R. (2003). Intervenciones psicoterapéuticas en un servicio de neonatología. Cuadernos de Psiquiatría y Psicoterapia del Niño y el Adolescente, 35-36, 169-181.

Tejedor, J.C., López de Heredia, J., Herranz, N., Nicolás, P., García-Muñoz, F. y Pérez-Rodríguez, J. Grupo de Trabajo de Ética de la Sociedad Española de Neonatología (2013). Recomendaciones sobre toma de decisiones y cuidados al final de la vida en neonatología. Anales de Pediatría, 78(1), 190.e1-190.e14.

Valle, M. (1991). Intervención en recién nacidos de alto riesgo biológico (Tesis Doctoral). Facultad de Pedagogía. Universidad Complutense. Madrid.

Valle, M. (2010). El Hospital de Día como dispositivo de apoyo para la evaluación e intervención en Atención Temprana. Cuadernos de Psiquiatría y Psicoterapia del niño y del adolescente, 50, 171-179.

Valle, M., Mateos, R. y Gutiez, P. (2012). Niños de Alto Riesgo al Nacimiento: Aspectos de Prevención. Atención Temprana Neonatal y Programas de Seguimiento en Niños Prematuros. Psicología Educativa,18, 135143. https://doi.org/10.5093/ed2012a14

Valle, M, Sierra, P. Palacios, Ormazábal, I. y Moro, M. (2014). Atención temprana al recién nacido de riesgo. Anales de Pediatría Continuada, 12(3), 119-123.

Artículo recibido: $15 / 04 / 2017$

Revisión recibida: 27/10/2017

Artículo aceptado: 30/10/2017 